\title{
Scattering in pseudo-Hermitian quantum field theory and causality violation
}

\author{
Oleg O. Novikov* \\ Saint Petersburg State University, 7/9 Universitetskaya nab., St. Petersburg 199034, Russia
}

(Received 22 January 2019; published 18 March 2019)

\begin{abstract}
The non-Hermitian but $\mathcal{P} \mathcal{T}$-symmetric quantum field theories are known to have a pseudo-Hermitian interpretation. However, the corresponding intertwining operator happens to be nonlocal that raises the question to what extent this nonlocality affects observable quantities. We consider the case when the intrinsic parity of the interaction terms is determined by degree of coupling constant. We show that the perturbative $\mathrm{S}$ matrix of the equivalent Hermitian description can be easily obtained from the perturbative S matrix of the non-Hermitian model. Namely, the first order vanishes whereas the second order is given by the real part of the second order $\mathrm{T}$ matrix of the non-Hermitian model. We compute directly the 2-point and 4-point correlation functions in the equivalent Hermitian model for the $i \phi^{3}$ model and find the results consistent with this relation. The 1-loop correction to the mass happens to be real reflecting the disappearance of 2-body decays. However, the 2 to 2 scattering amplitude obtained using LSZ formula has poles taken in principal value which implies the violation of the causality.
\end{abstract}

DOI: $10.1103 /$ PhysRevD.99.065008

\section{INTRODUCTION}

The non-Hermitian $\mathcal{P} \mathcal{T}$-symmetric quantum theories have attracted significant attention due to their unusual properties [1-3]. Surprisingly, they often possess purely real spectrum and produce an unitary evolution with respect to the nonstandard inner product. As a matter of fact, these Hamiltonians may be shown to be connected to the Hermitian Hamiltonians through the nonunitary intertwining operator and thus represent a particular class of the so-called pseudo-Hermitian Hamiltonians [4]. It is also interesting that some seemingly unstable Hamiltonians may be reinterpreted as non-Hermitian $\mathcal{P} \mathcal{T}$-symmetric Hamiltonians and then as pseudo-Hermitian Hamiltonians with purely real positive spectrum.

While most of the studies concentrated on the finitedimensional quantum mechanical models, the $\mathcal{P} \mathcal{T}$ symmetric quantum field theory (QFT) was also investigated [5-12]. This line of research is especially important because it gives a hope that some models interesting from a phenomenological perspective but plagued by unitarity and stability problems may be reinterpreted as consistent quantum field theories [13,14]. It inspired a number of proposals for the particle physics and cosmology [15-25].

\footnotetext{
*.novikov@spbu.ru
}

Published by the American Physical Society under the terms of the Creative Commons Attribution 4.0 International license. Further distribution of this work must maintain attribution to the author(s) and the published article's title, journal citation, and DOI. Funded by SCOAP .
However, many basic questions about the $\mathcal{P} \mathcal{T}$-symmetric quantum field theories that are important for their applicability have not yet been sufficiently investigated. One of such questions is whether these quantum field theories satisfy the relativistic causality. There is a very good reason to suspect that despite relativistic invariance, this may not be a case. As was shown in [6-8] for the $i \phi^{3}$ quantum field theory, the intertwining operator is nonlocal,

$$
\begin{aligned}
Q \simeq & \int d^{3} x d^{3} y d^{3} z[\mathcal{M}(\vec{x}, \vec{y}, \vec{z}) \pi(x) \pi(y) \pi(z) \\
& +\mathcal{N}(\vec{x} \mid \vec{y}, \vec{z}) \phi(y) \pi(x) \phi(z)] .
\end{aligned}
$$

As result the equivalent Hermitian Hamiltonian given by $h=e^{-Q / 2} H e^{Q / 2}$ is also nonlocal. This does not affect the locality of the vacuum correlators of the fields of the nonHermitian description that were extensively studied in the literature [5,9]. However, because such fields are not Hermitian under the modified norm, they are related to the observable fields through the same nonlocal intertwining operators. Therefore, one may expect the appearance of the causality violations.

In this paper, we are studying the $\mathrm{S}$ matrix in a nonHermitian $\mathcal{P} \mathcal{T}$-symmetric quantum field theory interpreted in a pseudo-Hermitian fashion. We present a very simple relation between $\mathrm{S}$-matrices in the non-Hermitian and equivalent Hermitian descriptions. However, this simple result shows the generic violation of the causality when the initial non-Hermitian model is local. We confirm this by directly computing the propagator and 2 to 2 scattering amplitude in the equivalent Hermitian model. 
It should be noted that the issue of nonlocality of the $\mathcal{P} \mathcal{T}$-symmetric quantum models with local non-Hermitian Hamiltonians arises already in the context of the finite dimensional quantum mechanical systems. Its influence on the scattering and the possibility to save the causality by relaxing the locality of the non-Hermitian Hamiltonian was studied in [26-29]. To our knowledge, our paper is the first one to address these questions in the context of the $\mathcal{P} \mathcal{T}$-symmetric QFT.

The paper is organized as follows. In Sec. II, we briefly elucidate the basics of the pseudo-Hermitian models. In Sec. III, we review the perturbation theory for the intertwining operator. In Sec. IV, this is used to compute the formal perturbative $\mathrm{S}$ matrix of the equivalent Hermitian model. In Sec. V, we derive the intertwining operator for the generic local $\mathcal{P} \mathcal{T}$-symmetric QFT in the momentum representation using a method that in our opinion is significantly simpler compared to the previous work. Section VI concludes this computation of the formal $\mathrm{S}$ matrix by showing that the commutator between the intertwining operators at large times vanishes. This leads to the extremely simple relation for the S-matrices of two equivalent descriptions; however, we also demonstrate the violation of the Bogolyubov microcausality. In Sec. VII, we formulate the simplest nontrivial $\mathcal{P} \mathcal{T}$-symmetric QFT, and in Sec. VIII, we develop a technique to compute correlation functions of the observable fields of the Hermitian model equivalent to this QFT. In Secs. IX and X, we apply this technique to the propagator and 2 to 2 scattering amplitude. In Sec. XI, we relax the assumption of the Hermiticity of the intertwining operator in an attempt to restore the causality. In the Conclusions, we summarize the results and discuss the prospects.

\section{II. $\mathcal{P} \mathcal{T}$-SYMMETRIC QUANTUM THEORY}

Consider the non-Hermitian but $\mathcal{P} \mathcal{T}$ Hamiltonian,

$$
H \neq H^{\dagger}, \quad[\mathcal{P} \mathcal{T}, H]=0,
$$

where $\mathcal{P}$ is the intrinsic parity operator (that reflects only fields and not spatial coordinates) and $\mathcal{T}$ is the usual time reflection operator. They are defined on the canonical fields $\phi(\vec{x})$ and their momenta $\pi(\vec{x})$ in the following way:

$$
\begin{aligned}
& \mathcal{P}: \phi(\vec{x}) \mapsto-\phi(\vec{x}), \quad \pi(\vec{x}) \mapsto-\pi(\vec{x}), \quad i \mapsto i \\
& \mathcal{T}: \phi(\vec{x}) \mapsto \phi(\vec{x}), \quad \pi(\vec{x}) \mapsto-\pi(\vec{x}), \quad i \mapsto-i,
\end{aligned}
$$

where the action on the imaginary unit means that $\mathcal{P}$ is a linear operator whereas $\mathcal{T}$ is antilinear. If one introduces the creation and annihilation operators,

$$
\phi(\vec{x})=\int \frac{d^{3} k}{(2 \pi)^{3}} \frac{1}{\sqrt{2 E_{\vec{k}}}}\left(a_{\vec{k}}^{\dagger} e^{-i \vec{k} \vec{x}}+a_{\vec{k}} e^{i \vec{k} \vec{x}}\right),
$$

TABLE I. The correspondence between the objects in the description with the non-Hermitian Hamiltonian and the equivalent Hermitian one.

\begin{tabular}{lcc}
\hline \hline Object & Non-Hermitian & Equivalent Hermitian \\
\hline Hamiltonian & $H$ & $h=\eta H \eta^{-1}$ \\
Inner product & $(\Psi, \Phi)=\left\langle\Psi\left|\eta^{\dagger} \eta\right| \Phi\right\rangle$ & $\langle\Psi \mid \Phi\rangle$ \\
Unobservable field & $\phi$ & $\eta \phi \eta^{-1}$ \\
Observable field & $\eta^{-1} \phi \eta$ & $\phi$ \\
\hline \hline
\end{tabular}

$$
\pi(\vec{x})=\int \frac{d^{3} k}{(2 \pi)^{3}} i \sqrt{\frac{E_{\vec{k}}}{2}}\left(a_{\vec{k}}^{\dagger} e^{-i \vec{k} \vec{x}}-a_{\vec{k}} e^{i \vec{k} \vec{x}}\right),
$$

the definitions above result in the following action:

$$
\mathcal{P} a_{\vec{k}} \mathcal{P}=-a_{\vec{k}}, \quad \mathcal{T} a_{\vec{k}} \mathcal{T}=a_{-\vec{k}} .
$$

As was shown in [1] such Hamiltonians often have purely real spectrum. This allows us to interpret them as a pseudo-Hermitian ones, i.e., related to some Hermitian Hamiltonian with the intertwining operator [4],

$$
h \equiv \eta H \eta^{-1}, \quad H^{\dagger} \eta^{\dagger} \eta=\eta^{\dagger} \eta H .
$$

The latter equation guarantees the Hermiticity of $h$ with respect to the initial product or equivalently the Hermiticity of $H$ with respect to the new inner product,

$$
(\Psi, \Phi) \equiv\left\langle\Psi\left|\eta^{\dagger} \eta\right| \Phi\right\rangle .
$$

We stress that this inner product is positively definite by construction, and thus, no negative norm states are needed. Note that the initial variable $\phi$ is not Hermitian under this inner product and thus is not observable. To get the observable that would correspond to the $\phi$ in the equivalent Hermitian description, one has to consider $\eta^{-1} \phi \eta$. In conclusion, there are two alternative description of the same model that are summarized in the Table I. One may note the trade-off between simplicity of the observables and simplicity of the evolution.

The relations above were written for time-independent $H$ in the Schrödinger picture. In the time-dependent case [30-34], the relation (8) is no longer true and an extra nonHermitian term appears. However, one can always define $\eta$ through the following property:

$\eta\left(t_{2}\right)^{\dagger} \eta\left(t_{2}\right) U_{H}\left(t_{2}, t_{1}\right)=\left[U_{H}\left(t_{2}, t_{1}\right)^{\dagger}\right]^{-1} \eta\left(t_{1}\right)^{\dagger} \eta\left(t_{1}\right)$,

where $U_{H}$ is the evolution operator generated by $H$. This relation simply means that the inner product $(\Psi, \Phi)$ is conserved by the temporal evolution of the state vectors and holds in all pictures.

Though this is not required for the Hermiticity of $h$, one usually assumes that the operator $\eta$ transforms in the 
following way so that the $\mathcal{P} \mathcal{T}$ symmetry of $H$ was conserved also by $h$ :

$$
\mathcal{P} \eta^{\dagger} \mathcal{P}=\eta^{-1}, \quad \mathcal{T} \eta^{\dagger} \mathcal{T}=\eta^{-1} .
$$

Also, one usually fixes $\eta$ to be Hermitian; thus, fixing the arbitrary unitary transformation one can insert into the intertwining operator. In this paper, we will hold to the assumptions of the Hermiticity and $\mathcal{P}$ parity but will not hold to the assumption of the $\mathcal{T}$ parity.

For the restricted class of Hamiltonians $\mathcal{P} H \mathcal{P}=H^{\dagger}$, the operator $\mathcal{C}$ is often introduced that have the following properties:

$\mathcal{C} \equiv \mathcal{P} \eta^{\dagger} \eta, \quad[\mathcal{C}, H]=0, \quad[\mathcal{C}, \mathcal{P} \mathcal{T}]=0, \quad \mathcal{C}^{2}=1$,

where all (11) are implied. Despite its notation, it is not related to the charge conjugation operator. The useful property of this operator is that $\mathcal{C}$ of an eigenstate of $H$ coincides with its $\mathcal{P} \mathcal{T}$ parity. Thus if the, e.g., the vacuum state $|\Omega\rangle$ is $\mathcal{P} \mathcal{T}$ symmetric then $\mathcal{C}|\Omega\rangle=1$ that significantly simplifies the computations on the vacuum state [35].

\section{PERTURBATION THEORY FOR $\boldsymbol{\eta}$}

Let us now assume that $H$ can be represented in the following form:

$$
\begin{aligned}
H & =\sum_{k=0}^{+\infty}(i g)^{k} H_{k}, \quad H_{k}=H_{k}^{\dagger}, \\
\mathcal{P} H_{k} \mathcal{P} & =(-1)^{k} H_{k}, \quad \mathcal{T} H_{k} \mathcal{T}=H_{k},
\end{aligned}
$$

where the coupling constant $g$ is assumed to be small. For purely imaginary coupling constants, the operator becomes Hermitian,

$$
\tilde{H}=\sum_{k=0}^{+\infty} g^{k} H_{k}
$$

We will assume that the corresponding evolution operators are also related by such analytic continuation at least within perturbation theory. That is if,

$$
U_{\tilde{H}}\left(t_{2}, t_{1}\right)=\mathrm{T} \exp \left[-i \int_{t_{1}}^{t_{2}} d t \tilde{H}\right]=\sum_{k=0}^{+\infty} g^{k} U_{H, k},
$$

we will assume that

$$
U_{H}\left(t_{2}, t_{1}\right)=\sum_{k=0}^{+\infty}(i g)^{k} U_{H, k} .
$$

We apply the similar decomposition to the equivalent Hermitian operator $h$ and to the intertwining operator $\eta$ (we omit the usual factor $1 / 2$ to simplify the equations), $h=\sum_{k=0}^{+\infty} g^{k} h_{k}, \quad \eta=\exp \left[-\sum_{k=0}^{+\infty} \frac{g^{2 k+1}}{(2 k+1) !} Q_{k}\right]$,

where $Q_{k}$ is assumed to have the following properties so that $\eta$ satisfied extra assumptions of Hermiticity and (11):

$$
Q_{k}^{\dagger}=Q_{k}, \quad\left\{Q_{k}, \mathcal{P}\right\}=0 .
$$

As with $\eta$, one may also demand

$$
\left\{Q_{k}, \mathcal{T}\right\}=0
$$

This makes it possible to choose the ansatz with only odd powers of $g$. As we will restrict ourselves only to the computations up to the second order in $g$, we will denote $Q \equiv Q_{0}$.

Then from (8), one obtains the following results [3]:

$$
i\left[H_{0}, Q\right]=H_{1},
$$

$$
h_{1}=0, \quad h_{2}=-H_{2}-\frac{i}{2}\left[Q, H_{1}\right] .
$$

To use (20), we note the following fact. As we work in the perturbation theory, let us go into the interaction picture,

$Q(t)=e^{i H_{0} t} Q e^{-i H_{0} t}, \quad H_{k}(t)=e^{i H_{0} t} H_{k} e^{-i H_{0} t}$.

Then (20) becomes a very simple relation,

$\partial_{t} Q(t)=H_{1}(t), \quad Q\left(t_{2}\right)-Q\left(t_{1}\right)=\int_{t_{1}}^{t_{2}} d t H_{1}(t)$.

We would like to stress that this relation can be obtained from the general equation for (10) written in the interaction picture. Thus, this results holds even if $H_{1}$ depends on $t$ explicitly, e.g., if one turns off interaction asymptotically.

No matter how simple (23) may appear, it has an important consequence. Even if the interaction falls down at large times, generally speaking the intertwining operator remains to be nontrivial. Thus, even though $H$ may appear Hermitian asymptotically, the field variables of the nonHermitian theory do not become observables and do not create good asymptotic particle states that are orthogonal with each other.

Thus, to understand the actual dynamics of the $\mathcal{P} \mathcal{T}$ symmetric QFT, one has to use either the non-Hermitian Hamiltonian $H$ and the modified field variables $\eta \phi(x) \eta^{-1}$ or equivalently, the Hermitian Hamiltonian $h$ and usual field variables. These two approaches should be fully equivalent, but in this paper, we choose the second one. 


\section{FORMAL $S$ MATRIX}

Let us consider the evolution operator of the equivalent Hermitian model in the interaction picture,

$$
U_{h}^{(I)}\left(t_{f}, t_{0}\right)=e^{i H_{0} t_{f}} e^{-i h\left(t_{f}-t_{0}\right)} e^{-i H_{0} t_{0}} .
$$

We can rewrite it in terms of the similar operator for the non-Hermitian Hamiltonian,

$$
\begin{aligned}
U_{h}^{(I)}\left(t_{f}, t_{0}\right) & =e^{i H_{0} t_{f}} \eta e^{-i H\left(t_{f}-t_{0}\right)} \eta^{-1} e^{-i H_{0} t_{0}} \\
& =\eta\left(t_{f}\right) U_{H}^{(I)} \eta\left(t_{0}\right)^{-1}
\end{aligned}
$$

where we define

$$
\eta(t) \equiv e^{i H_{0} t} \eta e^{-i H_{0} t} \simeq e^{-g Q(t)}+\mathcal{O}\left(g^{3}\right) .
$$

Let us now decompose the evolution operators into series in $g$,

$$
\begin{gathered}
U_{h}^{(I)} \simeq 1+g U_{h, 1}^{(I)}+g^{2} U_{h, 2}^{(I)}+\mathcal{O}\left(g^{3}\right) \\
U_{H}^{(I)} \simeq 1+i g U_{H, 1}^{(I)}-g^{2} U_{H, 2}^{(I)}+\mathcal{O}\left(g^{3}\right) .
\end{gathered}
$$

As noted above, we assume that the perturbation series of $U_{H}^{(I)}$ can be obtained by analytical continuation in $g$ of the perturbation series of the unitary operator $U_{\widetilde{H}}^{(I)}$. Then the unitarity implies

$$
U_{H, 1}=-\left(U_{H, 1}\right)^{\dagger}, \quad 2 \Re\left[U_{H, 2}\right]=\left[U_{H, 1}\right]^{2} .
$$

Using the Dyson expansion,

$$
U_{H, 1}^{(I)}\left(t_{f}, t_{0}\right)=-i \int_{t_{0}}^{t_{f}} d t H_{1}(t),
$$

and the relation (23), one finds,

$$
U_{h, 1}^{(I)}=-Q\left(t_{f}\right)+Q\left(t_{0}\right)+\int_{t_{0}}^{t_{f}} d t H_{1}(t)=0 .
$$

This should not come as a surprise because as we have noted in (21), the first order of $h$ vanishes.

For the second order, we get

$$
\begin{aligned}
U_{h, 2}^{(I)}= & -U_{H, 2}^{(I)}+\frac{Q^{2}\left(t_{f}\right)}{2}+\frac{Q^{2}\left(t_{0}\right)}{2}-Q\left(t_{f}\right) Q\left(t_{0}\right) \\
& -i Q\left(t_{f}\right) U_{H, 1}^{(I)}+i U_{H, 1}^{(I)} Q\left(t_{0}\right) .
\end{aligned}
$$

Again using (30) and (23), we rewrite it as

$$
U_{h, 2}^{(I)}=-U_{H, 2}^{(I)}-\frac{1}{2}\left[U_{H, 1}^{(I)}\right]^{2}+\frac{1}{2}\left[Q\left(t_{f}\right), Q\left(t_{0}\right)\right] .
$$

Finally using (29), we see that the second term cancels the real part of the first one,

$$
U_{h, 2}^{(I)}=-\Im\left[U_{H, 2}^{(I)}\right]+\frac{1}{2}\left[Q\left(t_{f}\right), Q\left(t_{0}\right)\right] .
$$

As usual, we apply the practical definition of the $\mathrm{S}$ matrix as the evolution operator in the interaction picture in the limit of large times (assuming that such limit exists),

$$
\begin{aligned}
& S_{h} \equiv \lim _{\substack{t_{f} \rightarrow+\infty \\
t_{0} \rightarrow-\infty}} U_{h}^{(I)}\left(t_{f}, t_{0}\right), \\
& S_{H} \equiv \lim _{\substack{t_{f} \rightarrow+\infty \\
t_{0} \rightarrow-\infty}} U_{H}^{(I)}\left(t_{f}, t_{0}\right) .
\end{aligned}
$$

Again assuming the validity of the validity of the analytical continuation from the unitary S matrix to $S_{H}$, we introduce the $\mathrm{T}$ matrix series in powers of $g$,

$$
\begin{aligned}
& S_{h} \simeq 1+i g T_{h}^{(1)}+i g T_{h}^{(2)}+\mathcal{O}\left(g^{3}\right), \\
& S_{H} \simeq 1-g T_{H}^{(1)}-i g T_{H}^{(2)}+\mathcal{O}\left(g^{3}\right) .
\end{aligned}
$$

Let us introduce the asymptotic intertwining operators,

$$
Q_{\text {in }}=\lim _{t \rightarrow-\infty} Q(t), \quad Q_{\text {out }}=\lim _{t \rightarrow+\infty} Q(t) .
$$

As the limit of (34), we obtain

$$
T_{h}^{(2)}=-\Re\left[T_{H}^{(2)}\right]-\frac{i}{2}\left[Q_{\mathrm{out}}, Q_{\mathrm{in}}\right] .
$$

\section{INTERTWINING OPERATOR IN QFT}

Let $H$ describe a $\mathcal{P} \mathcal{T}$-symmetric quantum field theory obtained through the analytical continuation in $g$ of the local quantum field theory.

$$
H_{1}(t)=\int d^{3} x V_{1}(t, \vec{x}), \quad \mathcal{P} V_{1} \mathcal{P}=-V_{1},
$$

$\mathcal{T} V_{1} \mathcal{T}=-V_{1}$.

Then, in the perturbation theory, one should be able to represent the interaction terms as combinations of the creation and annihilation operators,

$$
\begin{aligned}
H_{1}(t)= & \sum_{\left\{\varepsilon_{k}\right\}} \int \prod_{k} \frac{d^{3} p_{k}}{E_{\vec{p}_{k}}} \mathcal{V}_{\left\{\varepsilon_{k}\right\}}\left(\left\{\vec{p}_{k}\right\}\right) e^{i \sum_{k} \varepsilon_{k} E_{\overrightarrow{p_{k}}} t} \\
& \times \delta^{(3)}\left(\sum_{k} \varepsilon_{k} \vec{p}_{k}\right),
\end{aligned}
$$

where $\mathcal{V}$ is some operator valued distribution constructed as some $c$ function of $\left\{\varepsilon_{k}\right\}$ and $\left\{\vec{p}_{k}\right\}$ multiplied on a combination of the creation and annihilation operators in 
accordance with multiindex $\left\{\varepsilon_{k}\right\}$ so that $\varepsilon_{k}=+1$ corresponds to $a_{\vec{p}_{k}}^{\dagger}$ an $\varepsilon_{k}=-1$ corresponds to $a_{\vec{p}_{k}}$. The negative $\mathcal{P}$ parity means that only terms with odd number of these operators contribute.

The Hermiticity $H_{1}^{\dagger}=H_{1}$ means that

$$
\mathcal{V}_{\left\{\varepsilon_{k}\right\}}\left(\left\{\vec{p}_{k}\right\}\right)=\overline{\mathcal{V}}_{\sigma\left\{-\varepsilon_{k}\right\}}\left(\sigma\left\{\vec{p}_{k}\right\}\right),
$$

and $\mathcal{T} H_{1} \mathcal{T}=H_{1}$ yields

$$
\mathcal{V}_{\left\{\varepsilon_{k}\right\}}\left(\left\{\vec{p}_{k}\right\}\right)=\overline{\mathcal{V}}_{\left\{\varepsilon_{k}\right\}}\left(\left\{-\vec{p}_{k}\right\}\right),
$$

where $\overline{\mathcal{V}}$ denotes the complex conjugation of the $c$-numerical coefficients and $\sigma$ reverses the order in the multiindex.

We assume that a similar decomposition may be written for $Q(t)$,

$$
\begin{aligned}
Q(t)= & \sum_{\left\{\varepsilon_{k}\right\}} \int \prod_{k} \frac{d^{3} p_{k}}{E_{\vec{p}_{k}}} \mathcal{Q}_{\left\{\varepsilon_{k}\right\}}\left(\left\{\vec{p}_{k}\right\}\right) e^{i \sum_{k} \varepsilon_{k} E_{\overrightarrow{p_{k}}} t} \\
& \times \delta^{(3)}\left(\sum_{k} \varepsilon_{k} \vec{p}_{k}\right) .
\end{aligned}
$$

Then (23) yields the distribution equation,

$$
i\left[\sum_{k} \varepsilon_{k} E_{\vec{p}_{k}}\right] \mathcal{Q}_{\left\{\varepsilon_{k}\right\}}\left(\left\{\vec{p}_{k}\right\}\right)=\mathcal{V}_{\left\{\varepsilon_{k}\right\}}\left(\left\{\vec{p}_{k}\right\}\right),
$$

that has a general solution,

$$
\begin{aligned}
\mathcal{Q}_{\left\{\varepsilon_{k}\right\}}\left(\left\{\vec{p}_{k}\right\}\right)= & -\mathcal{V}_{\left\{\varepsilon_{k}\right\}}\left(\left\{\vec{p}_{k}\right\}\right) \text { P.v. } \frac{i}{\sum_{k} \varepsilon_{k} E_{\overrightarrow{p_{k}}}} \\
& +\mathcal{A}_{\left\{\varepsilon_{k}\right\}}\left(\left\{\vec{p}_{k}\right\}\right) \delta\left(\sum_{k} \varepsilon_{k} E_{\overrightarrow{p_{k}}}\right),
\end{aligned}
$$

where P.v. denotes the principal value and $\mathcal{A}$ is an arbitrary Hermitian operator value distribution. This reflects the freedom to add an arbitrary operator commuting with $H_{0}$. The most significant constraint comes from the Lorentz invariance of the inner product. We do not derive the most general form of $Q$ but instead simply choose

$$
\mathcal{A}_{\left\{\varepsilon_{k}\right\}}\left(\left\{\vec{p}_{k}\right\}\right)=\alpha \mathcal{V}_{\left\{\varepsilon_{k}\right\}}\left(\left\{\vec{p}_{k}\right\}\right) .
$$

This simple ansatz results in

$$
\begin{aligned}
Q(t)= & -i \sum_{\left\{\varepsilon_{k}\right\}} \int \prod_{k} \frac{d^{3} p_{k}}{E_{\vec{p}_{k}}} \mathcal{V}_{\left\{\varepsilon_{k}\right\}}\left(\left\{\vec{p}_{k}\right\}\right) \\
& \times\left[\text { P.v. } \frac{e^{i \sum_{k} \varepsilon_{k} E_{\overrightarrow{p_{k}}} t}}{\sum_{k} \varepsilon_{k} E_{\overrightarrow{p_{k}}}} \delta^{(3)}\left(\sum_{k} \varepsilon_{k} \vec{p}_{k}\right)\right. \\
& \left.+i \alpha \delta^{(4)}\left(\sum_{k} \varepsilon_{k} p_{k}\right)\right] .
\end{aligned}
$$

Such $Q$ obviously has the same $\mathcal{P}$ parity as $H_{1}$. From (43) and the evenness of the $\delta$ function follows that the Hermiticity of $Q$ requires $\alpha=\alpha^{*}$. This is exactly what we need if we want to shift the pole in the fraction in the complex plane. However (44) and the symmetry of the second term under reflection of $\vec{p}_{k}$ means that for (19) to be true one needs $\alpha=-\alpha^{*}$. Thus, to shift the pole, one has to relax this extra assumption and thus break $\mathcal{P} \mathcal{T}$ symmetry of $h$.

One may check that (49) indeed conserves the Lorentz invariance of the inner product (9). The operator of the Lorentz boost $\Lambda$ characterized by rapidity $\vec{\beta}$ may be written as

$$
\mathcal{U}_{g}(\Lambda) \simeq 1+i \beta_{i}\left(L_{0}^{0 i}(t)+i g L_{1}^{0 i}(t)\right)
$$

where $L_{0}^{0 i}$ is the boost operator in the free QFT and,

$$
\begin{aligned}
L_{1}^{0 i}(t)= & -\int d^{3} x x^{i} V_{1}(t, \vec{x}) \\
= & i \sum_{\left\{\varepsilon_{k}\right\}} \int \prod_{k} \frac{d^{3} p_{k}}{E_{\vec{p}_{k}}} \nabla_{i}^{(P)}\left[\mathcal{V}_{\left\{\varepsilon_{k}\right\}}\left(\left\{\vec{p}_{k}\right\}\right)\right] e^{i \sum_{k} \varepsilon_{k} E_{\overrightarrow{p_{k}}} t} \\
& \times \delta^{(3)}\left(\sum_{k} \varepsilon_{k} \vec{p}_{k}\right)
\end{aligned}
$$

where we define the total momentum as

$$
\vec{P}=\sum_{k} \varepsilon_{k} \vec{p}_{k}, \quad P^{0}=\sum_{k} \varepsilon_{k} E_{\vec{p}_{k}} .
$$

We assume that $V_{1}(t, \vec{x})$ represented using interaction picture fields transforms as a Lorentz scalar field with respect to the boost of the free QFT. As in (42), we used the Lorentz invariant integration measure $\mathcal{V}$ transforms as a Lorentz scalar too,

$$
\left[\mathcal{U}_{0}(\Lambda)\right]^{-1} \mathcal{V}_{\left\{\varepsilon_{k}\right\}}\left(\left\{\vec{p}_{k}\right\}\right) \mathcal{U}_{0}(\Lambda)=\left.\mathcal{V}_{\left\{\varepsilon_{k}\right\}}\left(\left\{\Lambda^{-1} \vec{p}_{k}\right\}\right)\right|_{p_{k}^{0}=E_{\vec{p}_{k}}}
$$

For an infinitesimal boost $\Lambda^{-1} \vec{p}_{k} \simeq \vec{p}_{k}-\vec{\beta} E_{\vec{p}_{k}}$, this implies

$$
\left[L_{0}^{0 i}(t), \mathcal{V}_{\left\{\varepsilon_{k}\right\}}\left(\left\{\vec{p}_{k}\right\}\right)\right]=i P^{0} \nabla_{i}^{(P)}\left[\mathcal{V}_{\left\{\varepsilon_{k}\right\}}\left(\left\{\vec{p}_{k}\right\}\right)\right]
$$

Combining this with (49) and (51), one easily obtains that the inner product (9) is indeed Lorentz invariant,

$$
\begin{array}{rl}
{[\mathcal{U}(\Lambda)]^{\dagger} \eta^{\dagger} \eta \mathcal{U}(\Lambda)-\eta^{\dagger} \eta \simeq 2} & 2 i g \beta_{i}\left(\left[L_{0}^{0 i}(t), Q(t)\right]\right. \\
& \left.+i L_{1}^{0 i}(t)\right)=0 .
\end{array}
$$

This confirms and generalizes the result obtained in [8]. Indeed, in case of the $i \phi^{3}$ model, if $\alpha=0$, (49) gives the 
same nonlocal intertwining operator (1) as the one studied in $[6-8]$.

\section{FORMAL $S$ MATRIX IN $\mathcal{P} \mathcal{T}$-SYMMETRIC QFT}

To find $Q_{\text {out }}$ and $Q_{\text {in }}$, we first explicitly compute $T_{H}^{(1)}$ by integrating (42) and using the standard integral representation of the $\delta$ function,

$$
\begin{aligned}
T_{H}^{(1)} & =2 \pi \sum_{\left\{\varepsilon_{k}\right\}} \int \prod_{k} \frac{d^{3} p_{k}}{E_{\vec{p}_{k}}} \mathcal{V}_{\left\{\varepsilon_{k}\right\}}\left(\left\{\vec{p}_{k}\right\}\right) \delta^{(4)}\left(\sum_{k} \varepsilon_{k} p_{k}\right), \\
p_{k}^{0} & \equiv E_{\vec{p}_{k}},
\end{aligned}
$$

we use the following identity [36] in a sense of a distribution on localized wave packets in $E$ [that usually is represented by shifting $t_{f} \mapsto t_{f}(1-i \epsilon)$ in the QFT textbooks],

$$
\text { P.v. } \frac{e^{i E t}}{E}=\frac{1}{2} \frac{e^{i E t}}{E+i \epsilon}+\frac{1}{2} \frac{e^{i E t}}{E-i \epsilon} \underset{t \rightarrow \pm \infty}{\longrightarrow} \pm \pi i \delta(E) .
$$

This yields

$$
Q_{\text {out }}=\frac{\pi+\alpha}{2 \pi} T_{H}^{(1)}, \quad Q_{\text {in }}=\frac{-\pi+\alpha}{2 \pi} T_{H}^{(1)} .
$$

This result makes the violation of $\mathcal{T}$-symmetry by nonzero $\alpha$ easily recognizable. One can also easily see that

$$
\left[Q_{\text {out }}, Q_{\text {in }}\right]=0,
$$

and thus (40) simplifies to

$$
T_{h}^{(2)}=-\mathfrak{R}\left[T_{H}^{(2)}\right]
$$

One may then easily show that if $H_{1}$ is the local Hamiltonian the $\mathrm{S}$ matrix of the equivalent Hermitian theory will almost always lead to the causality violation. For example, the Bogolyubov microcausality condition requires [37],

$$
\frac{\delta}{\delta \phi_{k}(z)}\left(\frac{\delta S}{\delta \phi_{j}(y)} S^{\dagger}\right) \sim \theta\left(z^{0}-y^{0}\right) \theta\left((z-y)^{2}\right),
$$

This condition means that if we represent the evolution of the wave functional as a sequence of the scattering events of the localized wave packets, the secondary wave packets will be produced only in the future light cones of regions of the intersections of the primary wave packets. This guarantees that both ordinary causality (future does not influence the past) and the relativistic causality (no superluminal propagation) holds.

If $H_{1}$ is given by (41) and $H_{2}$ is also a local operator represented as a integral of $V_{2}(x)$ then using (60), we obtain

$$
\begin{aligned}
T_{h}^{(2)}= & -\int d^{4} x V_{2}(x)+\frac{i}{4} \int d^{4} x_{1} d^{4} x_{2} \varepsilon\left(x_{1}^{0}-x_{2}^{0}\right) \\
& \times\left[V_{1}\left(x_{1}\right), V_{2}\left(x_{2}\right)\right] .
\end{aligned}
$$

Then we get

$\frac{\delta}{\delta \phi_{k}(z)}\left(\frac{\delta S_{h}}{\delta \phi_{j}(y)} S_{h}^{\dagger}\right) \simeq \frac{g^{2}}{2} \varepsilon\left(y^{0}-z^{0}\right)\left[\frac{\partial V_{1}}{\partial \phi_{j}}(y), \frac{\partial V_{1}}{\partial \phi_{k}}(z)\right]$,

that is nonzero not only in the future light cone but also in the past light cone. Therefore, the secondary wave packets are produced in both the future and the past light cones of the intersection regions of the primary wave packets. This obviously violates the ordinary causality. In a sequence of collisions, this also leads to the superluminal propagation; however, there is possibility that this violation of the relativistic causality is somehow compensated in the higher orders of the $\mathrm{S}$ matrix. The obvious exception is the linear potential $V_{1}(x)=a \phi(x)$. Then the operator $\eta$ becomes simply a shift of the field variable in the imaginary direction that may be reabsorbed into other interactions without violation of locality.

\section{EXAMPLE OF THE $\mathcal{P} \mathcal{T}$-SYMMETRIC QFT}

Let us consider the model with the following action:

$$
\begin{aligned}
S= & \int d^{4} x\left[\frac{1}{2} \partial_{\mu} \phi_{k} \partial^{\mu} \phi_{k}-\frac{m_{k}^{2}}{2}\left(\phi_{k}\right)^{2}-i g \frac{\mu_{i j k}}{3 !} \phi_{i} \phi_{j} \phi_{k}\right. \\
& \left.-g^{2} \frac{\lambda_{i j k l}}{4 !} \phi_{i} \phi_{j} \phi_{k} \phi_{l}\right]
\end{aligned}
$$

where all coupling constants are assumed to be real and totally symmetric under permutations of indices. As usual, we use the canonical momenta of the free theory $\pi_{k}(\vec{x}) \equiv$ $\dot{\phi}_{k}(\vec{x})$ that may be represented as (6). Then the first few terms of the Hamiltonian in the (13) become

$$
\begin{gathered}
H_{0}=\int d^{3} x\left[\frac{1}{2}\left(\pi_{k}\right)^{2}+\frac{1}{2}\left(\nabla \phi_{k}\right)^{2}+\frac{m^{2}}{2}\left(\phi_{k}\right)^{2}\right], \\
H_{1}=\int d^{3} x\left[\frac{\mu_{i j k}}{3 !} \phi_{i} \phi_{j} \phi_{k}+\delta_{t, k} \phi_{k}\right], \\
H_{2}=\int d^{3} x\left[\frac{\lambda_{i j k l}}{4 !} \phi_{i} \phi_{j} \phi_{k} \phi_{l}+\frac{\delta_{m, j k}}{2} \phi_{j} \phi_{k}\right. \\
\left.+\frac{\delta_{Z, j k}}{2}\left(\pi_{j} \pi_{k}-\left(\nabla \phi_{j} \cdot \nabla \phi_{k}\right)\right)\right]
\end{gathered}
$$

where we introduced the counterterms (also symmetric under permutations of indices) to absorb the divergences and use more sensible renormalized perturbation theory. 
Because of the way $H_{2}$ contributes to $h_{2}$ in (21) in the process up to the second order of $g^{2}$, the $\phi^{4}$ term gives a contribution only as a local interaction term. Thus, we will set for the rest of the paper $\lambda_{i j k l}=0$. However, in higher orders, one needs to take into account both the $\phi^{4}$ term and its counterterm.

From now on, we will represent all operators in the interaction picture. We will use the following definition for the Feynman propagators and related functions:

$$
\begin{gathered}
D_{F}^{(j k)}(x-y) \equiv\left\langle 0\left|\mathrm{~T}\left\{\phi_{j}(x) \phi_{k}(y)\right\}\right| 0\right\rangle, \\
\left.\left.\left.\bar{D}_{F}^{(j k)}(x-y) \equiv\langle 0| \overline{\mathrm{T}}\left\{\phi_{j}(x) \phi_{k}(y)\right]\right\} \mid 0\right]\right\rangle \\
D^{(j k)}(x-y) \equiv\left[\phi_{j}(x), \phi_{k}(y)\right]=\varepsilon\left(x^{0}\right)\left(D_{F}^{(j k)}(x)-\bar{D}_{F}^{(j k)}(x)\right),
\end{gathered}
$$

$$
\tilde{D}^{(j k)}(x-y) \equiv D_{F}^{(j k)}(x-y)+\bar{D}_{F}^{(j k)}(x-y),
$$

where $\mathrm{T}$ and $\overline{\mathrm{T}}$ are chronological and antichronological products, respectively,

$$
\begin{aligned}
\mathrm{T}\left\{\phi_{j}(x) \phi_{k}(y)\right\}= & \theta\left(x^{0}-y^{0}\right) \phi_{j}(x) \phi_{k}(y) \\
& +\theta\left(y^{0}-x^{0}\right) \phi_{k}(y) \phi_{j}(x), \\
\overline{\mathrm{T}}\left\{\phi_{j}(x) \phi_{k}(y)\right\}= & \theta\left(x^{0}-y^{0}\right) \phi_{k}(y) \phi_{j}(x) \\
& +\theta\left(y^{0}-x^{0}\right) \phi_{j}(x) \phi_{k}(y) .
\end{aligned}
$$

Because all these functions are proportional to the $\delta_{j k}$ symbol, we will also use $D^{(k)}(x) \equiv D^{(k k)}(x)$. This yields the following momentum representation:

$$
\begin{gathered}
D(x-y)=\int \frac{d^{4} p}{\left(2 \pi^{4}\right.} D(p) e^{-i p(x-y)} \\
D_{F}^{(k)}(p)=\frac{i}{p^{2}-m_{k}^{2}+i \epsilon}, \quad \bar{D}_{F}^{(k)}(p)=-\frac{i}{p^{2}-m_{k}^{2}-i \epsilon} \\
D^{(k)}(p)=\frac{2 \pi}{2 E_{\vec{p}}^{(k)}}\left(\delta\left(p^{0}-E_{\vec{p}}^{(k)}\right)-\delta\left(p^{0}+E_{\vec{p}}^{(k)}\right)\right)
\end{gathered}
$$

where $E_{\vec{p}}^{(k)} \equiv \sqrt{m_{k}^{2}+\vec{p}^{2}}$.

We now may consider the following correlation functions:

$$
\begin{aligned}
\mathcal{G}_{H, n}(1, \ldots n) & \equiv\left\langle\Omega\left|\mathrm{T}\left\{\phi_{i_{1}}\left(x_{1}\right) \ldots \phi_{i_{n}}\left(x_{n}\right)\right\}\right| \Omega\right\rangle_{H} \\
& =\frac{\left\langle 0\left|\mathrm{~T}\left\{\phi_{i_{1}}\left(x_{1}\right) \ldots \phi_{i_{n}}\left(x_{n}\right) S_{H}\right\}\right| 0\right\rangle}{\left\langle 0\left|S_{H}\right| 0\right\rangle} .
\end{aligned}
$$

We assume that they can be obtained by simple analytical continuation of the correlation functions in the Hermitian $\phi^{3}$ model to the complex values of the coupling constant (this was argued to be valid in [5] in contrast to the similar approach to the $-g^{2} \phi^{4}$ model plagued by the breakdown of the ordinary perturbation theory). It is interesting that because with extra assumption (19) the metric operator $\eta^{\dagger} \eta$ happens to act trivially on the interacting vacuum $|\Omega\rangle$, the inner product in this correlation function may be understood as the modified inner product $(\Psi, \Phi)$ and thus is conserved [35]. This was used extensively to study the path integral of the non-Hermitian model. However, it is not clear what relation these correlation functions have to observables as $\phi(x)$ are not Hermitian with respect to the $(\Psi, \Phi)$. Also because $\phi(x)$ do not commute with $\eta^{\dagger} \eta$, this trick is not particularly helpful for the scattering processes.

The easiest counterterm to take into account is the tadpole counterterm. It represents the shift of the field, and in the Hermitian sigma model, it is usually fixed so that the field was zero in the vacuum,

$$
\langle\Omega|\phi(x)| \Omega\rangle_{H}=0,
$$

that yields that the tadpole absorbs the singular term without any extra regular part,

$$
\delta_{t, k}=-\frac{\mu_{k j j}}{2} D_{F}^{(j)}(0) .
$$

This may also be rewritten as the statement that the interaction Hamiltonian is normally ordered,

$$
H_{1}=\int d^{3} x\left[: \frac{\mu_{i j k}}{3 !} \phi_{i} \phi_{j} \phi_{k}:\right]
$$

In the pseudo-Hermitian model, we could add the real regular part to $\delta_{t, k}$. Unlike the Hermitian case, the shift of the field would be done in the imaginary axis and would become a part of the intertwining operator $Q$. This would be equivalent to certain shifts of the masses and coupling constants. To not overcomplicate things, we set the tadpole counterterm to (79).

Then we renormalize the propagator,

$$
\Pi_{H}^{\left(i_{1} i_{2}\right)}(p)=\int d^{4}(\Delta x) e^{i p \Delta x} \mathcal{G}_{H, 2}(1,2) .
$$

In the perturbation theory, we can represent it as

$$
\Pi_{H}^{\left(i_{1} i_{2}\right)} \simeq D_{F}^{\left(i_{1} i_{2}\right)}+i g^{2} D_{F}^{\left(i_{1}\right)} \Delta_{H}^{i_{1} i_{2}} D_{F}^{\left(i_{2}\right)}
$$

where

$$
\Delta_{H}^{i_{1} i_{2}}=-\frac{i}{2} \mu_{i_{1} j k} \mu_{i_{2} j k} I_{j k}(p)+\delta_{m, i_{1} i_{2}}-\delta_{Z, i_{1} i_{2}} p^{2}
$$




$$
I_{j k}(p)=\int \frac{d^{4} k}{(2 \pi)^{4}} D_{F}^{(j)}(k) D_{F}^{(k)}(p-k) .
$$

Counterterms $\delta_{m, i j}$ and $\delta_{Z, i j}$ are used to absorb the divergent part of this integral that depends on the choice of the regularization, substraction scheme, and renormalization conditions. Our results do not depend on this choices but to satisfy the assumptions (13), we assume that the counterterms are real.

\section{CORRELATION FUNCTIONS IN THE EQUIVALENT HERMITIAN QFT}

We now are interested in computation of the correlation functions in the equivalent Hermitian model using the standard formula,

$$
\begin{aligned}
\mathcal{G}_{n}(1 \ldots n) & \equiv\left\langle\Omega\left|\mathrm{T}\left\{\phi_{i_{1}}\left(x_{1}\right) \ldots \phi_{i_{n}}\left(x_{n}\right)\right\}\right| \Omega\right\rangle \\
& =\frac{\left\langle 0\left|\mathrm{~T}\left\{\phi_{i_{1}}\left(x_{1}\right) \ldots \phi_{i_{n}}\left(x_{n}\right) S_{h}\right\}\right| 0\right\rangle}{\left\langle 0\left|S_{h}\right| 0\right\rangle},
\end{aligned}
$$

where $S_{h}$ is understood as in (35). To compute it, we use the Dyson series and the relation (21),

$S_{h} \simeq 1-i g^{2} \int_{-\infty}^{+\infty} d t\left[-H_{2}(t)+\frac{i}{2}\left[H_{1}(t), Q(t)\right]\right]$.

As $H_{2}(t)$ is the integral of the local operator, we may compute its contribution in a standard way. The real challenge is in the computation of the contribution of the commutator. To compute it, we remember (23) to represent it as

$$
\left[H_{1}(t), Q(t)\right]=\left.\partial_{\tau}^{-1}\left[H_{1}(t), H_{1}(\tau)\right]\right|_{\tau=t},
$$

where we define the antiderivative according to our choices in Sec. V,

$$
\partial_{\tau}^{-1} e^{i E t} \equiv-i e^{i E t}\left[\mathrm{P} . \mathrm{v} \cdot \frac{1}{E}+i \alpha \delta(E)\right] .
$$

As in most practical QFT computations, we will assume that all our operators are sufficiently nice operator valued distributions and this operation commutes with all integrals except integrals in $t$ and $\tau$. Also, we will assume that it commutes with the operation of taking the vacuum expectation value.

Taking the local operator representation of $H_{1}(t)$,

$$
H_{1}(t)=\int d^{3} w V(w), \quad w^{0} \equiv w,
$$

we represent the contribution of the commutator to the $\mathrm{S}$ matrix as

$$
\begin{aligned}
\delta S_{h} & =-i g^{2} \int_{-\infty}^{+\infty} d t \frac{i}{2}\left[H_{1}(t), Q(t)\right] \\
& =-i g^{2} \int d^{4} z d^{4} w \delta\left(w^{0}-z^{0}\right) \partial_{w^{0}}^{-1} \mathcal{W}(z, w),
\end{aligned}
$$

where

$$
\mathcal{W}(z, w)=\frac{i}{2}[V(z), V(w)]
$$

Finally, with all our assumptions we write the contribution to the correlation function as

$\delta \mathcal{G}_{n}(1 \ldots n)=-i g^{2} \int d^{4} z d^{4} w \delta\left(w^{0}-z^{0}\right) \partial_{w^{0}}^{-1} \mathcal{F}_{n}(1 \ldots n \mid z, w)$,

where we introduced

$$
\begin{aligned}
\mathcal{F}_{n}(1 \ldots n \mid z, w) \equiv & \left\langle 0\left|\mathrm{~T}\left\{\phi_{i_{1}}\left(x_{1}\right) \ldots \phi_{i_{n}}\left(x_{n}\right) \mathcal{W}(z, w)\right\}\right| 0\right\rangle \\
& -\left\langle 0\left|\mathrm{~T}\left\{\phi_{i_{1}}\left(x_{1}\right) \ldots \phi_{i_{n}}\left(x_{n}\right)\right\}\right| 0\right\rangle \\
& \times\langle 0| \mathcal{W}(z, w)\}|0\rangle .
\end{aligned}
$$

To use that formula, all we need is to represent $\mathcal{W}(z, w)$ in terms of the chronological product. To do so we first rewrite it as

$\left.\mathcal{W}(z, w)=\frac{i}{2} \varepsilon\left(z^{0}-w^{0}\right) \cdot[\mathrm{T}\{V(z) V(w)\}-\overline{\mathrm{T}}\{V(z) V(w)\}]\right]$.

We will consider the model from the previous section, i.e.,

$$
V(x)=: \frac{\mu_{i j k}}{3 !} \phi_{i}(x) \phi_{j}(x) \phi_{k}(x): .
$$

Then we use the Wick's theorem for the chronological product and a similar statement for the antichronological product (that works by simply replacing $D_{F} \mapsto \bar{D}_{F}$ ) to convert (94) to the normal form. The normal ordering of $V$ means that we should not include the terms with contractions of fields with same coordinates. As result we get

$$
\begin{aligned}
\mathcal{W}(z, w)= & \frac{i}{8} \mu_{i j k} \mu_{l m k}\left[: \phi_{i}(z) \phi_{j}(z) \phi_{l}(w) \phi_{m}(w): D^{(k)}(z-w)\right. \\
& +2: \phi_{i}(z) \phi_{l}(w): D^{(k)}(z-w) \tilde{D}^{(j m)}(z-w) \\
& +(c \text {-number })] .
\end{aligned}
$$

We do not track the $c$ number as its contribution is canceled out by the vacuum normalization. Then, we use the Wick's theorem again to rewrite it as 


$$
\begin{aligned}
\mathcal{W} & (z, w) \\
= & \frac{i}{8} \mu_{i j k} \mu_{l m k}\left[+\mathrm{T}\left\{: \phi_{i}(z) \phi_{j}(z):: \phi_{l}(w) \phi_{m}(w):\right\} D^{(k)}(z-w)\right. \\
& -4 \mathrm{~T}\left\{\phi_{i}(z) \phi_{l}(w)\right\} D^{(k)}(z-w) D_{F}^{(j m)}(z-w) \\
& +2 \mathrm{~T}\left\{\phi_{i}(z) \phi_{l}(w)\right\} D^{(k)}(z-w) \tilde{D}^{(j m)}(z-w) \\
& +(c \text {-number })] .
\end{aligned}
$$

\section{HERMITIAN FIELD PROPAGATOR}

The propagator is computed according to the standard formula,

$$
\Pi^{\left(i_{1} i_{2}\right)}(p)=\int d^{4}(\Delta x) e^{i p \Delta x} \mathcal{G}_{2}(1,2)
$$

For the 2-point contribution of $\mathcal{W}$ we obtain

$$
\begin{aligned}
\mathcal{F}(1,2 \mid z, w)= & \frac{i}{4} \mu_{i_{1} j k} \mu_{i_{2} j k} D^{(k)}(z-w) \tilde{D}_{F}^{(j)}(z-w) \\
& \times\left[D_{F}^{\left(i_{1}\right)}\left(x_{1}-z\right) D_{F}^{\left(i_{2}\right)}\left(x_{2}-w\right)\right. \\
& \left.+D_{F}^{\left(i_{1}\right)}\left(x_{1}-w\right) D_{F}^{\left(i_{2}\right)}\left(x_{2}-z\right)\right] .
\end{aligned}
$$

Using our choice of (88), we find that the first term gives the following contribution to the propagator:

$$
\begin{aligned}
\delta \Pi_{1}^{\left(i_{1} i_{2}\right)}(p)= & -\frac{i g^{2}}{4} \mu_{i_{1} j k} \mu_{i_{2} j k} \int d^{4}(\Delta x) \int d z^{0} \int d^{3} z d^{3} w \int \frac{d^{4} p_{1}}{(2 \pi)^{4}} \frac{d^{4} p_{2}}{(2 \pi)^{4}} \frac{d^{4} q}{(2 \pi)^{4}} \frac{d^{4} r}{(2 \pi)^{4}} \\
& \times e^{i\left(p-p_{1}\right) \Delta x-i\left(p_{1}+p_{2}\right) x_{2}} e^{i\left(p_{1}^{0}+p_{2}^{0}\right) z^{0}-i \vec{p}_{1} \vec{z}-i \vec{p}_{2} \vec{w}+i(\vec{q}+\vec{r})(\vec{z}-\vec{w})} D_{F}^{\left(i_{1}\right)}\left(p_{1}\right) D_{F}^{\left(i_{2}\right)}\left(p_{2}\right) \tilde{D}_{F}^{(j)}(q) D_{F}^{(k)}(r) \\
& \times\left[\text { P.v. } \frac{1}{q^{0}+l^{0}+p_{2}^{0}}+i \alpha \delta\left(q^{0}+l^{0}+p_{2}^{0}\right)\right] .
\end{aligned}
$$

Integrals over $\Delta x, z^{0}, \vec{z}$, and $\vec{w}$ introduce the $\delta$ functions that fix the momenta, and using the momentum representation of $D^{(k)}$ in (76), we obtain

$$
\begin{aligned}
\delta \Pi_{1}^{\left(i_{1} i_{2}\right)}(p)= & -\frac{i g^{2}}{4} \mu_{i_{1} j k} \mu_{i_{2} j k} D_{F}^{\left(i_{1}\right)}(p) D_{F}^{\left(i_{2}\right)}(p) \int \frac{d^{4} q}{(2 \pi)^{4}} \tilde{D}_{F}^{(j)}(q)\left[-\mathrm{P} \cdot \mathrm{v} \frac{1}{(p-q)^{2}-m_{k}^{2}}\right. \\
& \left.+i \alpha \varepsilon\left(p^{0}-q^{0}\right) \delta\left((p-q)^{2}-m_{k}^{2}\right)\right] .
\end{aligned}
$$

Similar computation for the contribution of the second term in (99) yields

$$
\begin{aligned}
\delta \Pi_{2}^{\left(i_{1} i_{2}\right)}(p)= & -\frac{i g^{2}}{4} \mu_{i_{1} j k} \mu_{i_{2} j k} D_{F}^{\left(i_{1}\right)}(p) D_{F}^{\left(i_{2}\right)}(p) \int \frac{d^{4} q}{(2 \pi)^{4}} \tilde{D}_{F}^{(j)}(q)\left[-\mathrm{P} \cdot \mathrm{v} \frac{1}{(p-q)^{2}-m_{k}^{2}}\right. \\
& \left.-i \alpha \varepsilon\left(p^{0}-q^{0}\right) \delta\left((q-p)^{2}-m_{k}^{2}\right)\right] .
\end{aligned}
$$

Thus, the $\alpha$ term cancels out. As the counterterms give the standard contribution, the full propagator can be written in the standard way,

$$
\Pi^{\left(i_{1} i_{2}\right)} \simeq D_{F}^{\left(i_{1} i_{2}\right)}+i g^{2} D_{F}^{\left(i_{1}\right)} \Delta^{\left(i_{1} i_{2}\right)} D_{F}^{\left(i_{2}\right)}
$$

where

$$
\begin{aligned}
\Delta^{\left(i_{1} i_{2}\right)}= & \frac{1}{2} \mu_{i_{1} j k} \mu_{i_{2} j k}\left(\Im\left[I_{j k}(p)\right]+\Im\left[\tilde{I}_{j k}(p)\right]\right) \\
& +\delta_{m, i_{1} i_{2}}-\delta_{Z, i_{1} i_{2}} p^{2}
\end{aligned}
$$

where $I_{j k}(p)$ is given by (84) and
$\tilde{I}_{j k}(p)=-\int \frac{d^{4} q}{(2 \pi)^{4}} \frac{1}{q^{2}-m_{j}^{2}+i \epsilon} \frac{1}{(p-q)^{2}-m_{k}^{2}-i \epsilon}$.

This loop integral is problematic as the standard Wick rotation fails, and its accurate computation may significantly depend on the regularization. The issues with the Wick rotation issues are characteristic for the nontrivial QFT setting, e.g., on curved backgrounds or in the noncommutative geometry models $[38,39]$. However, we present a simple symmetry argument that hopefully allows us to omit this integral altogether. We note that under $j \leftrightarrow k$ these integrals transform as

$$
I_{j k}(p)=I_{k j}(p), \quad \tilde{I}_{j k}(p)=\tilde{I}_{k j}^{*} .
$$




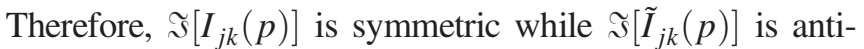
symmetric. Therefore, the latter does not make any contribution. Thus,

$$
\begin{aligned}
\Delta^{\left(i_{1} i_{2}\right)} & =\frac{1}{2} \mu_{i_{1} j k} \mu_{i_{2} j k} \Im\left[I_{j k}(p)\right]+\delta_{m, i_{1} i_{2}}-\delta_{Z, i_{1} i_{2}} p^{2} \\
& =\Re\left[\Delta_{H}^{\left(i_{1} i_{2}\right)}\right],
\end{aligned}
$$

where in the end we used the reality of the counterterms. This is consistent with our general formula (60). The most interesting aspect of this result is that if non-Hermitian model admitted a two body, some of $\Delta_{H}^{\left(i_{1} i_{2}\right)}$ would get the imaginary part corresponding to the decay width. However, because $h_{1}=0$, the equivalent Hermitian model never admits any two body decays. Thus, according to the optical theorem, to keep unitarity, $\Delta^{\left(i_{1} i_{2}\right)}$ must remain real, and indeed, this is what happens.

\section{2 TO 2 SCATTERING AMPLITUDE}

To find a 2 to 2 scattering amplitude, we first separate the connected part of the 4-point correlation function,

$$
\begin{aligned}
\mathcal{G}_{4}(1,2,3,4)= & \mathcal{G}_{4, c}(1,2,3,4)+\mathcal{G}_{2}(1,2) \mathcal{G}_{2}(3,4) \\
& +\mathcal{G}_{2}(1,3) \mathcal{G}_{2}(2,4)+\mathcal{G}_{2}(1,4) \mathcal{G}_{2}(2,3)
\end{aligned}
$$

To compute the $\mathcal{G}_{4, c}$, one needs to include only the terms where all external fields $\phi_{i_{k}}\left(x_{k}\right)$ are contracted with fields in $\mathcal{W}$. That means that only the 4-field term in (97) gives contribution. It can be further separated into three scattering channels,

$$
\begin{aligned}
\mathcal{G}_{4, c}(1,2,3,4)= & \tilde{\mathcal{G}}_{4}(1,2 \mid 3,4)+\tilde{\mathcal{G}}_{4}(1,3 \mid 2,4) \\
& +\tilde{\mathcal{G}}_{4}(1,4 \mid 2,3), \\
\tilde{\mathcal{G}}_{4, c}(1,2 \mid 3,4)= & -i g^{2} \int d^{4} z d^{4} w \delta\left(w^{0}-z^{0}\right) \\
& \times \partial_{w^{0}}^{-1} \tilde{\mathcal{F}}_{4}(1,2|3,4| z, w) .
\end{aligned}
$$

For each scattering channel, we get

$$
\begin{aligned}
\tilde{\mathcal{F}}_{4}(1,2|3,4| z, w)= & \frac{i}{2} \mu_{i_{1} i_{2} k} \mu_{i_{3} i_{4} k} D^{(k)}(z-w)\left[D_{F}^{\left(i_{1}\right)}\left(x_{1}-z\right)\right. \\
& \times D_{F}^{\left(i_{2}\right)}\left(x_{2}-z\right) D_{F}^{\left(i_{3}\right)}\left(x_{3}-w\right) \\
& \left.\times D_{F}^{\left(i_{4}\right)}\left(x_{4}-w\right)+((1,2) \leftrightarrow(3,4))\right] .
\end{aligned}
$$

In the momentum representation,

$$
\begin{aligned}
\hat{\tilde{\mathcal{G}}}_{4, c}(1,2 \mid 3,4) \equiv & \int \prod_{k}\left\{d^{4} x_{k} e^{i p_{k} x_{k}}\right\} \tilde{\mathcal{G}}_{4, c}(1,2 \mid 3,4) \\
= & \frac{g^{2}}{2} \mu_{i_{1} i_{2} k} \mu_{i_{3} i_{4} k} D_{F}^{i_{1}}\left(p_{1}\right) D_{F}^{i_{2}}\left(p_{3}\right) D_{F}^{i_{4}}\left(p_{3}\right) \\
& \times D_{F}^{i_{4}}\left(p_{4}\right)\left[A^{(k)}\left(p_{1}+p_{2} \mid p_{3}+p_{4}\right)\right. \\
& \left.+A^{(k)}\left(p_{3}+p_{4} \mid p_{1}+p_{2}\right)\right],
\end{aligned}
$$

where we introduced

$$
\begin{aligned}
A^{(k)}\left(p_{\text {in }} \mid p_{\text {out }}\right)= & -i \int d z^{0} d^{3} z d^{3} w \int \frac{d^{4} q}{(2 \pi)^{4}} D^{(k)}(q) \\
& \times e^{i\left(p_{\text {in }}^{0}+p_{\text {out }}^{0}\right) z^{0}+i \vec{q}(\vec{z}-\vec{w})-i \vec{p}_{\text {in }} \vec{z}-i \vec{p}_{\text {out }} \vec{w}} \\
& \times\left[\text { P.v. } \frac{1}{p_{\text {out }}^{0}-q^{0}}+i \alpha \delta\left(p_{\text {out }}^{0}-q^{0}\right)\right] .
\end{aligned}
$$

The integrals over $z$ and $w$ result in $\delta$ functions for momenta, and using the momentum representation of $D^{(k)}$ in (76), we obtain

$$
\begin{aligned}
A^{(k)}\left(p_{\text {in }} \mid p_{\text {out }}\right)= & -i(2 \pi)^{4} \delta^{(4)}\left(p_{\text {in }}+p_{\text {out }}\right)\left[\text { P.v. } \frac{1}{\left(p_{\text {out }}\right)^{2}-m_{k}^{2}}\right. \\
& \left.+i \alpha \varepsilon\left(p_{\text {out }}^{0}\right) \delta\left(\left(p_{\text {out }}\right)^{2}-m_{k}^{2}\right)\right] .
\end{aligned}
$$

Because the $\alpha$ term happens to be antisymmetric under the $p_{\text {in }} \leftrightarrow p_{\text {out }}$ exchange, it cancels out, and we get

$$
\begin{aligned}
\hat{\tilde{\mathcal{G}}}_{4, c}(1,2 \mid 3,4)= & -i(2 \pi)^{4} \delta^{(4)}\left(\sum_{n} p_{n}\right) g^{2} \mu_{i_{1} i_{2} k} \mu_{i_{3} i_{4} k} D_{F}^{i_{1}}\left(p_{1}\right) \\
& \times D_{F}^{i_{2}}\left(p_{2}\right) D_{F}^{i_{3}}\left(p_{3}\right) D_{F}^{i_{4}}\left(p_{4}\right) \\
& \times \text { P.v. } \frac{1}{\left(p_{3}+p_{4}\right)^{2}-m_{k}^{2}} .
\end{aligned}
$$

Let us apply the standard LSZ formula for the 2 to 2 scattering amplitude,

$$
\begin{aligned}
\hat{\mathcal{G}}_{4, c}(1,2,3,4) \underset{\text { on-shell }}{\sim} & (2 \pi)^{4} \delta^{(4)}\left(p_{1}+p_{2}+p_{1}+p_{2}\right) D_{F}^{\left(i_{1}\right)}\left(p_{1}\right) \\
& \times D_{F}^{\left(i_{2}\right)}\left(p_{2}\right) D_{F}^{\left(i_{3}\right)}\left(q_{1}\right) D_{F}^{\left(i_{4}\right)}\left(q_{2}\right) \\
& \times \mathcal{A}_{2 \rightarrow 2}^{\left(i_{1} i_{2} \mid i_{3} i_{4}\right)}\left(p_{1}, p_{2} \mid-p_{3},-p_{4}\right) ;
\end{aligned}
$$

we obtain a simple result, 


$$
\begin{aligned}
& \mathcal{A}_{2 \rightarrow 2}^{\left(i_{1} i_{2} \mid j_{1} j_{2}\right)}\left(p_{1}, p_{2} \mid q_{1}, q_{2}\right) \\
& =-i g^{2} \mu_{i_{1} i_{2} k} \mu_{j_{1} j_{2} k}\left[\text { P.v. } \frac{1}{s-m_{k}^{2}}+\text { P.v. } \frac{1}{t-m_{k}^{2}}+\text { P.v. } \frac{1}{u-m_{k}^{2}}\right],
\end{aligned}
$$

where $(s, t, u)$ are the standard Mandelstam variables. One may recognize the scattering amplitude of the $\phi^{3}$ theory analytically continued to the imaginary coupling. However, the poles are taken in principal value. This is in a perfect agreement with the general formula (60).

However, this simple result has a significant problem. The scattering amplitude (117) violates the well-known causality constraints on the analytical structure of the scattering amplitude [40,41]. In fact, we can represent the amplitude above as

$$
\mathcal{A}_{2 \rightarrow 2}=-\frac{1}{2} \mathcal{A}_{2 \rightarrow 2}^{(+i \epsilon)}+\frac{1}{2} \mathcal{A}_{2 \rightarrow 2}^{(-i \epsilon)}
$$

The first term corresponds to the 2 to 2 scattering amplitude in the Hermitian $\phi^{3}$ QFT that respects the causal nature of the scattering - the scattered particles are produced only in the future light cone of the collision event of the ingoing particles. On the other hand, the second term respects the reversed causality - the scattered particles are produced only in the past light cone of the collision event of the ingoing particles. Thus, the total scattering amplitude is acausal in a full accordance with (63). One could conjecture that this happens because of the extra restriction (19) and may be avoided by a $\mathcal{T}$-violating extra term that shifts the poles in (88) to the lower complex half-plane. However, as we have shown, the result happened to not depend on $\alpha$. Indeed among the extra assumptions in our derivation of (60), we used only (18) but not (19).

\section{ATTEMPT AT RELAXING THE HERMITICITY ASSUMPTION}

One may conjecture that the equivalent Hermitian theory may actually be causal but the field operators obtained with use of the Hermitian intertwining operator correspond to nonlocal configurations. Then there may exist an unitary transformation that makes the causal nature of the model apparent. While not proving that such operator does not exist, we show how the simplest ansatz of this sort fails.

Let us take the Hermitian $Q$ satisfying (23) but modify the formula for the intertwining operator,

$$
\eta=e^{-g(1+i \theta) Q}+\mathcal{O}\left(g^{3}\right) .
$$

Then the metric operator $\eta^{\dagger} \eta$ remains to be the same, and therefore such intertwining operator converts a nonHermitian theory into the Hermitian one.
Repeating the computations from Secs. III and IV, one obtains the following equivalent Hermitian Hamiltonian:

$h^{(\theta)} \simeq H_{0}+g \theta H_{1}+g^{2}\left(-H_{2}+\frac{1+\theta^{2}}{2}\left[H_{1}, Q\right]\right)$

and the corresponding formal $\mathrm{S}$ matrix looks like

$S_{h}^{(\theta)} \simeq 1+i g \theta T_{H}^{(1)}+i g^{2}\left(-T_{H}^{(2)}+\frac{i}{2}\left(1+\theta^{2}\right)\left[T_{H}^{(1)}\right]^{2}\right)$.

Using the results of the previous section, it is trivial to confirm that the 2 to 2 scattering amplitude computed for $h^{(\theta)}$ is in agreement with the above formula for $S_{h}^{(\text {theta) }}$ and can be written as

$$
\begin{aligned}
\mathcal{A}_{2 \rightarrow 2}^{\left(i_{1} i_{2} \mid j_{1} j_{2}\right)}\left(p_{1}, p_{2} \mid q_{1}, q_{2}\right) & \\
= & -i g^{2} \mu_{i_{1} i_{2} k} \mu_{j_{1} j_{2} k}\left[\text { P.v. } \frac{1}{s-m_{k}^{2}}+i \pi \theta \delta\left(s-m_{k}^{2}\right)\right] \\
& +(t \text { and } u \text { terms }) .
\end{aligned}
$$

Sadly, $\theta$ pushes the pole in the wrong direction. The most curious case is when $\theta=1$. Then, the first order $T$ matrix coincides with a first order $T$ matrix of the Hermitian $\phi^{3}$ model. However, while the propagator remains to have the right ie prescription, the 2 to 2 scattering amplitude is analytical in the wrong complex half-plane. Thus, this simple ansatz can not help us restore causality.

\section{CONCLUSIONS}

In this work, we have considered the perturbative scattering in the local non-Hermitian $\mathcal{P} \mathcal{T}$-symmetric quantum field theory interpreted in a pseudo-Hermitian fashion. We explicitly showed that the intertwining operator remains to be nontrivial even when the interactions asymptotically vanishes. In fact, the intertwining operator in the limit of large times is proportional to the first order T matrix of the non-Hermitian model. This makes it quite hard to associate the field variables of the initial non-Hermitian model with any actual observables even in the asymptotic region. Thus, we resort to the computations with equivalent Hermitian Hamiltonian.

Despite this equivalent Hamiltonian being quite complicated and nonlocal, the surprising result is that the leading order of its $\mathrm{S}$ matrix is very simply related to the $\mathrm{S}$ matrix of the original non-Hermitian model. The generic consequences of this relation include the disappearance of two body decay that are also reflected in the 1-loop correction to the mass of particles but also the violation of the causal analytic structure of the 2 to 2 body scattering amplitude. These effects were explicitly 
demonstrated for the $i \phi^{3}$ model. Our result raise a question whether the causality violation in such models may prevent possible applications from being phenomenologically viable.

The modifications of the analytic structure of the propagators that destroy the ordinary notion of the microscopic causality were studied before in the context of the models with indefinite metrics or $\mathcal{C P} \mathcal{T}$ violation [42-44]. As a matter of fact, our results bear strong similarity to the models of the so-called shadow states [41,45]. While nowhere in our consideration, the indefinite norm appears (see [46] and references therein for the discussion on the positivity of the norm in the $\mathcal{P} \mathcal{T}$-symmetric models), one may conjecture that the pseudo-Hermitian interpretation of the local $\mathcal{P} \mathcal{T}$-symmetric QFT may be equivalent to the positive norm sector of some QFT with indefinite metrics. At this level, this conjecture remains to be purely hypothetical and will be explored in the future work.

As was noted in [27-29], the causality issues of the finite dimensional $\mathcal{P} \mathcal{T}$-symmetric quantum mechanics may be tackled by introducing certain degree of nonlocality into the non-Hermitian Hamiltonian. Similar strategy may work in case of QFT, and the simple form of our results may imply that a certain modified notion of locality of the non-Hermitian Hamiltonian may arise.

In the end of Sec. VI, we mentioned that the case of the linear potential is exceptional and preserves causality. This happens because the intertwining operator becomes simply a shift of the field variable in the imaginary direction and thus preserves the local structure of the Hamiltonian. Because we restricted ourselves to the lowest orders of perturbation theory about free bosonic QFT with polynomial interactions, this case may appear to be somewhat trivial. However in [47], it was shown that the shift intertwining operator can be used for the nonperturbative construction of the $\mathcal{P} \mathcal{T}$-symmetric SineGordon model.

We conclude by reviewing several shortcomings one may see in our consideration.

(i) The results presented in this paper are derived with a level of rigor typical for the practical QFT computations that involves rather liberal operations with integrals of the operator-valued distributions and no discussions of the domain issues and existence of operators in question beyond the perturbation theory level. It should be stressed however that this sloppy (though highly fruitful) approach is characteristic also to the field of the $\mathcal{P} \mathcal{T}$-symmetric quantum theories even when finite-dimensional quantum mechanics is considered. In the opinion of the author, many questions about the Hilbert spaces involved and existence of the intertwining operator in a strict sense (not just on the Hamiltonian eigenstates but also on a generic quantum state) are yet to be addressed. Thus, we deem it premature to consider the $\mathcal{P} \mathcal{T}$-symmetric $\mathrm{QFT}$ on a more rigorous level and are prepared for the loopholes associated with singular transformations of a generic wave functional even within the realm of the perturbation theory.

(ii) As we mentioned in Sec. XI, the causality problem may be simply an artifact of choosing the bad variables, and some unitary transformation may fix it. We were not able to do so by choosing the simplest ansatz, but it may be possible to find the more appropriate one by studying accurately the asymptotic conditions. Our results were also restricted to the lowest nontrivial order with computations in the higher orders being much more nontrivial.

(iii) Throughout the paper, we assumed the applicability of the perturbation theory as it is presented in Sec. III. The acausal poles may actually signify its breakdown and may disappear after an appropriate resummation. The applicability of the perturbation theory for the formal correlation functions in the $\mathcal{P} \mathcal{T}$-symmetric non-Hermitian QFT was studied in [5]. But again for the correlation functions of the equivalent Hermitian model, we have no simple form beyond the lowest order and thus leave this important question for the future investigation.

(iv) As we mentioned above, the solution (49) for the intertwining operator that has a smooth momentum representation and does not break the Lorentz invariance is not unique because an arbitrary integral of motion of the free QFT can be added. This may greatly improve the locality of the resulting equivalent Hermitian Hamiltonian [48]. Our general formula (40) implies that for this to work the commutator $\left[Q_{\text {out }}, Q_{\text {in }}\right]$ must not vanish. One may give a specific example in case of the fermionic QFT considered in [47]. In that case, one may construct the quadratic non-Hermitian interaction satisfying the parity conventions (13),

$$
\mathcal{L}=\bar{\psi}\left(i \gamma^{\mu} \partial_{\mu}-m_{1}-m_{2} \gamma_{5}\right) \psi .
$$

Interestingly, the aforementioned paper presents the local intertwining operator in the form,

$$
Q \sim \int d x \psi^{\dagger} \gamma_{5} \psi,
$$

whereas the ansatz similar to (49) would produce a different highly nonlocal intertwining operator,

$$
Q \sim \int d x \bar{\psi} \gamma_{5}\left(-i \gamma^{k} \partial_{k}+m\right)^{-1} \psi .
$$

However, one may see many differences with the bosonic case considered in this paper. With bosonic 
fields, $H_{1}$ should contain the odd number of fields to have the appropriate $\mathcal{P}$ parity. More so the spinor field rotation does not introduce derivative terms into the interaction Hamiltonian because $\Psi^{\dagger}$ serves as a canonical momentum for $\Psi$ as opposed to $\dot{\phi}$ in the bosonic case. Thus, the fermionic $\mathcal{P} \mathcal{T}$-symmetric QFT may be more well behaved and deserves additional analysis. At this point, it is not clear whether a good $Q$ that preserves both causality and Lorentz invariance exists for bosonic field interactions.

\section{ACKNOWLEDGMENTS}

The author would like to thank A. A. Andrianov, M. V. Ioffe, M. A. Braun, A. V. Golovnev, and M. A. Kurkov for helpful discussions and support. The funding for this work was provided by the RFBR Project No. 18-02-00264.
[1] C. M. Bender and S. Boettcher, Phys. Rev. Lett. 80, 5243 (1998).

[2] A. A. Andrianov, F. Cannata, J. P. Dedonder, and M. V. Ioffe, Int. J. Mod. Phys. A 14, 2675 (1999).

[3] C. M. Bender, Rep. Prog. Phys. 70, 947 (2007).

[4] A. Mostafazadeh, J. Math. Phys. (N.Y.) 43, 205 (2002).

[5] C. M. Bender, K. A. Milton, and V. M. Savage, Phys. Rev. D 62, 085001 (2000).

[6] C. M. Bender, D. C. Brody, and H. F. Jones, Phys. Rev. D 70, 025001 (2004); 71, 049901(E) (2005).

[7] C. M. Bender, D. C. Brody, and H. F. Jones, Phys. Rev. Lett. 93, 251601 (2004).

[8] C. M. Bender, S. F. Brandt, J.-H. Chen, and Q.-h. Wang, Phys. Rev. D 71, 065010 (2005).

[9] C. M. Bender, N. Hassanpour, S.P. Klevansky, and S. Sarkar, Phys. Rev. D 98, 125003 (2018).

[10] J. Alexandre, P. Millington, and D. Seynaeve, Phys. Rev. D 96, 065027 (2017).

[11] J. Alexandre, J. Ellis, P. Millington, and D. Seynaeve, Phys. Rev. D 98, 045001 (2018).

[12] J. Alexandre, J. Ellis, P. Millington, and D. Seynaeve, Phys. Rev. D 98, 045001 (2018).

[13] C. M. Bender, S. F. Brandt, J.-H. Chen, and Q.-h. Wang, Phys. Rev. D 71, 025014 (2005).

[14] C. M. Bender, D. W. Hook, N. E. Mavromatos, and S. Sarkar, J. Phys. A 49, 45LT01 (2016).

[15] J. Alexandre, C. M. Bender, and P. Millington, J. Phys. Conf. Ser. 873, 012047 (2017).

[16] J. Alexandre, C. M. Bender, and P. Millington, J. High Energy Phys. 11 (2015) 111.

[17] T. Ohlsson, Europhys. Lett. 113, 61001 (2016).

[18] P. D. Mannheim, Found. Phys. 42, 388 (2012).

[19] A. A. Andrianov, F. Cannata, and A. Y. Kamenshchik, Int. J. Mod. Phys. D 15, 1299 (2006).

[20] A. A. Andrianov, F. Cannata, and A. Y. Kamenshchik, J. Phys. A 39, 9975 (2006).

[21] A. A. Andrianov, C. Lan, and O. O. Novikov, Springer Proc. Phys. 184, 29 (2016).

[22] O. O. Novikov, Phys. Part. Nucl. Lett. 15, 353 (2018).

[23] O. Novikov, EPJ Web Conf. 191, 07005 (2018).

[24] M. A. Braun and G. P. Vacca, Eur. Phys. J. C 50, 857 (2007).
[25] M. A. Braun and G. P. Vacca, Eur. Phys. J. C 59, 795 (2009).

[26] H. F. Jones, Phys. Rev. D 76, 125003 (2007).

[27] M. Znojil, Phys. Rev. D 78, 025026 (2008).

[28] M. Znojil, J. Phys. A 41, 292002 (2008).

[29] M. Znojil, Phys. Rev. D 80, 045009 (2009).

[30] A. Mostafazadeh, Phys. Lett. B 650, 208 (2007).

[31] F. Cannata, M. V. Ioffe, G. Junker, and D. Nishnianidze, J. Phys. A 32, 3583 (1999).

[32] A. Fring and M. H. Y. Moussa, Phys. Rev. A 93, 042114 (2016).

[33] M. Znojil, Phys. Rev. D 78, 085003 (2008).

[34] M. Znojil, Acta Polytech. 50, 62 (2010).

[35] H. F. Jones and R. J. Rivers, Phys. Rev. D 75, 025023 (2007).

[36] S.S. Schweber, An Introduction to Relativistic Quantum Field Theory (Row, Peterson and Company, Evanston, Illinois, and Elmsford, New York, 1961), p. 321.

[37] N. N. Bogolubov, A. A. Logunov, A. I. Oksak, and I. T. Todorov, General Principles of Quantum Field Theory (Kluwer Academic Publishers, Dordrecht, Boston, London, 1990), p. 537.

[38] M. Visser, arXiv:1702.05572.

[39] F. D’Andrea, M. A. Kurkov, and F. Lizzi, Phys. Rev. D 94, 025030 (2016).

[40] R. J. Eden, P. V. Landshoff, D. I. Olive, and J. C. Polkighorne, The Analytic S-Matrix (Cambridge University Press, Cambridge, England, 1966), pp. 204-220.

[41] H. Rechenberg and E. C. G. Sudarshan, Nuovo Cimento A 14, 299 (1973).

[42] N. Nakanishi, Prog. Theor. Phys. Suppl. 51, 1 (1972).

[43] M. A. Kurkov and V. A. Franke, Found. Phys. 41, 820 (2011).

[44] M. Chaichian, K. Fujikawa, and A. Tureanu, Eur. Phys. J. C 73, 2349 (2013).

[45] C. A. Nelson and E. C. G. Sudarshan, Phys. Rev. D 6, 3658 (1972).

[46] A. Mostafazadeh, Czech. J. Phys. 53, 1079 (2003).

[47] C. M. Bender, H. F. Jones, and R. J. Rivers, Phys. Lett. B 625, 333 (2005).

[48] The author would like to thank the referee for this observation and pointing out an example. 